\title{
A vinculação dos conhecimentos: entre a razão mediada e a razão leve $^{1}$
}

\author{
Maria Nelida Gonzalez de Gómez*
}

Resumo Durante séculos, existiam poucas dúvidas acerca da validade e da qualidade dos princípios que colocavam juntos conhecimentos, textos, documentos, conteúdos disciplinares e suas respectivas literaturas. No século XX, não se poderia manter esse otimismo, confrontado com a pluralidade e a diversificação das especialidades, assim como a fragilização das fronteiras entre as ciências e as não-ciências e entre disciplinas. A questão, agora, seria acerca da extensão e da qualidade dessas mudanças e do entendimento de seu significado na esfera social.

Palavras-chave Conhecimento, informação, vinculações.

\begin{abstract}
For centuries, there has been little doubt about the validity and the nature of the principles that put together knowledge, texts, documents, disciplinary subject matters and the respective literatures. In the twentieth century, this optimistic assumption is confronted by the plurality and diversification of specialities, and by the weakening of boundaries between sciences and non-sciences, and between disciplines. The question, now, is about the extension of these changes and the understanding of its significance for social spheres.
\end{abstract}

Keywords Knowledge, information, links.

\section{Introdução}

Neste trabalho, entenderemos por organização do conhecimento o modo como os conhecimentos se relacionam e se diferenciam, nas práticas de sua produção e uso (GONZALEZ DE GOMEZ, 1996).

Os discursos acadêmicos e os jornalísticos são prolíferos hoje na tematização da produção e da organização dos conhecimentos, considerados tanto como "eixos" quanto indicadores de grandes

\footnotetext{
${ }^{1}$ Este trabalho faz parte da contribuição da autora aos projetos de pesquisa "Vinculações: as novas formas de vinculação entre conhecimentos, discursos, sujeitos e seus efeitos na recuperação e busca de informação", projeto desenvolvido com auxílio do $\mathrm{CNPq}$, sob sua coordenação; e o projeto "Memória e identidade: a construção discursivo-metafórica nas novas tecnologias da informação", projeto desenvolvido com auxílio do CNPq, coordenado por Evelyn Goyannes Dill Orrico, professora e coordenadora do Programa de Pós-Graduação em Memória Social e Documento, Uni-Rio, da qual e colaboradora; ao mesmo tempo, somos reconhecidas das imprescindíveis oportunidades de reflexão, critica e informação que nos foi proporcionada durante todo o desenvolvimento dos dois projetos.
}

Doutora em Comunicação. Pesquisadora Titular IBICT-MCT. Professora do Programa de Pos- Graduação em Ciência da informação (UFF-IBICT). Email: nelida@ibict.br 
mudanças, de modo que as transformações manifestas em seus domínios seriam generalizáveis como indicadores de mudanças sociais da máxima extensão, tal como quando se fala de Sociedade da Informação ou Sociedade do Conhecimento.

Entre essas mudanças, teríamos novos modos de interação e coordenação entre as disciplinas e especialidades assim como entre as esferas da ciência e as esferas econômicas e políticas.

A fragilização das fronteiras (POMBO, 2004) seria, de fato, um dos principais assuntos a serem debelados.

As palavras com que se tematizam as questões fazem parte do sentido da própria questão: interdisciplinaridade, transdisciplinaridade, disciplinas pós-acadêmicas, modos de conhecimento 1 e 2 remetem "a reformulação das fronteiras do conhecimento científico - e sua redistribuição em espaços acadêmicos e econômicos, públicos e privados; hibridismo; transversalidade, transgressão, passagem, translação são expressões igualmente usadas nos estudos da ciência e da cultura. Um outro conjunto de discursos significativos analisa as modalidades de colaboração entre cientistas, atores econômicos e agencias governamentais; muitos outros abordam as novas tecnologias interativas e a hipermídia do ponto de vista de sua intervenção na produção compartilhada ou distribuída dos conhecimentos e nas trocas de informação.

Se, como conceitos ou noções menos formalizadas, tais expressões identificam autores, linhas de pensamento e domínios de pesquisa, como sintomas ficam ancorados nos contextos espaçotemporais de sua manifestação - ainda que as duas séries não se relacionem entre si de maneira biunívoca. Não existiriam, porém, rupturas nítidas entre uma epistemologia política e um uso político da epistemologia e de categorias gnosiológicas.

Conforme Wallerstein (1995), criar fronteiras em torno de "setores" implica uma decisão social com conseqüências na distribuição de poder e de recursos. "E o que foi criado pela sociedade, pode ser anulado pela sociedade” (WALLERSTEIN, 1995, p. 340).

Segmentações, fronteiras e vínculos entre conhecimentos são resultado, concordamos, das maneiras históricas e situadas com que os homens, ao relacionar-se entre si e com o mundo, tecem a teia de saberes e discursos em que tais relações são construídas e "tematizadas".

Para tratar de entender o significado e alcance do que hoje se caracteriza como mudança na forma de produzir e transmitir conhecimentos, revisaremos o conceito de especialidade, lembraremos um processo que denominamos de "opacificação" da ciência para acompanhar sua objetivação e reconstrução integradora ora pelo controle administrativo de sua produtividade, ora pelo "externalismo" metodológico dos estudos sociais e antropológicos. Sem pretensões de "palavra final", recorreremos a algumas das propostas alternativas de interdisciplinaridade, transdisciplinaridade e de transversalidade, deixando em aberto a reflexão sobre a figura e a condição da racionalidade que se manifesta à luz daqueles novos cenários.

\section{Os pontos de partida: disciplinas e especialidades}

Um ponto de partida iniludível desta reflexão seria os conceitos de disciplina e de especialidade. Para Morin, é próprio da disciplina buscar sua autonomia: a) pela delimitação de suas fronteiras; b) pela constituição de uma linguagem própria; c) pelas técnicas que elabora ou utiliza; e c) pela 
construção de conceitos e teorias. "Uma disciplina pode ser definida como uma categoria organizadora do conhecimento científico: ela institui a divisão e especialização do trabalho, e responde à diversidade dos domínios que recobrem as ciências" (MORIN, 2000, p.65).

A história das disciplinas teria, porém, duas versões: a oficial e a não-oficial, onde acontecem trocas, movimentos de importação e exportação de conceitos, procedimentos, informações. Se uma disciplina diferencia-se das outras pelo ponto de vista diferencial, pelo qual vai configurar seu objeto como algo "extraído ou construído por processos específicos", deverá manter-se atenta ao campo de visão, espaço ideal de reconstrução das relações que religam seu objeto a outros saberes disciplinares e a outros domínios de objetos, que mantêm sempre vital e atualizada a agenda disciplinar. A suspensão desse duplo movimento de fechamento e abertura da disciplina levaria à "cosificação" de seu objeto e à sua estagnação.

Para Lenoir (AMARAL, 1997), a institucionalização da ciência na universidade moderna teria contribuído para a legitimação das grades classificatórias disciplinares que regulam a produção formalizada dos conhecimentos. Tais disciplinas teriam o caráter de estruturas socioculturais cuja finalidade seria reunir, reproduzir e orientar as práticas sociais e técnicas que os sistemas econômico e de poder consideram necessárias a seu funcionamento. Como disciplinas, a Física e a Biologia não seriam assim puras manifestações da diversidade gnosiológica, mas figuras de políticas epistemológicas da sociedade, a configurar-se através das grandes áreas disciplinares.

Para diversos autores, existiria uma diferença entre a disciplina e a pesquisa (CHUBIN, 1976; LATOUR, 1998). A pesquisa nunca contribuiria ao desenvolvimento de uma totalidade disciplinar, mas faria aportes só a algumas de seus aspectos, questões ou temáticas (CHUBIN, 1976, p. 448). Essas unidades menores, micro-ambientes da pesquisa, conforme definição anterior de Hagstrom (1970, p. 93 apud CHUBIN, 1976, p. 448), seriam as especialidades, as quais constituem uma unidade social e intelectual (onde estariam fortemente associados uma rede de relações estruturadas dos pesquisadores e o desenvolvimento intelectual da especialidade), unidade sustentada por uma rica rede de comunicação, de modo que a densidade das relações entre os membros da especialidade será sempre maior que a densidade das relações entre os que são membros da especialidade e os que não o são. Tal enredamento de práticas e discursos dentro da especialidade, mas com movimentos de migração de pessoas, procedimentos e instrumentos, justifica a utilização dos modelos de redes para seu estudo. ${ }^{2}$

Cabe então perguntamo-nos: qual seria a diferença entre essas dinâmicas de "reprodução ampliada" das especialidades e as configurações designadas como inter- e transdisciplinares?

Em primeiro lugar, a sociologia das especialidades partiu de uma concepção "internalista" da ciência, não dando atenção aos ambientes institucionais, técnicos e administrativos onde acontecem as pesquisas. Em segundo lugar, desenvolveu um modelo hierárquico-centralizado da pesquisa, representado pela figura núcleo-periferia, com um centro identitário que manteria a autoridade científica como principio legitimador, subordinando outros aportes à cancela da especialidade preferencial. ${ }^{3}$

Não pareceria, porém, que essa descrição fosse adequada para as configurações do conhecimento que se caracterizam como inter ou transdiciplinares, ou ainda híbridas. A "inteligência artificial", por exemplo, designaria um pool de conhecimentos que inclui a lógica, as matemáticas, a física, as ciências cognitivas, a lingüística computacional e as ciências computacionais. Esses

\footnotetext{
${ }^{2}$ Chubin (1976) cita, entre outros, Granovetter e Kadushin.

${ }^{3}$ Os estudos das especialidades deram assim visibilidade às dinâmicas da produção dos conhecimentos, reconhendo que seus movimentos de expansão e renovação regulada admitem estados de expansão progressista ou estagnação (LAKATOS, 1998)
} 
entrançados de saberes, que provêm de diversas áreas especializadas, não formam, porém, uma estrutura fixa núcleo-periferia; ao menos em certos momentos ou programas de pesquisa, uma ou outra subárea pode ocupar uma posição de destaque e poderia rearranjar as relações de hierarquia e interdependência. Sempre, porém, em malhas relativamente frouxas, sem que seja possível prever qual será o próximo realinhamento dos saberes participantes (RAMMERT, 2004).

\section{Heterogeneidade dos discursos e das práticas das ciências e o controle exteriorizado dos conhecimentos}

Um dos primeiros indicadores das mudanças que se manifestariam na reformulação dos modos de vinculação entre os conhecimentos é o que denominaremos a "opacificação da ciência" e a afirmação da heterogeneidade das práticas e discursos qualificados como científicos.

Kuhn, pós-empiricista, atuando no campo da História das Ciências, em sincronia com a teoria dos jogos da linguagem de Wittgenstein, elabora o conceito de "paradigma", no qual leva a suas últimas conseqüências os efeitos epistêmicos das especialidades, afirmando o caráter local e contextual dos discursos e práticas científicas. Para Kuhn, diferentes matrizes disciplinares agem como diferentes culturas ou subculturas, incomensuráveis em seus modos de olhar e de nomear suas objetivações construídas, de modo que não se poderia manter a diferenciação entre conceitos teóricos e conceitos empíricos. Entre um e outro paradigma, ou entre um e outro jogo de linguagem não há tradução, mas "conversão".

Para Foucault, a enunciação da pluralidade e da heterogeneidade dos conhecimentos, incluídos os científicos, não seria tanto uma descoberta ou descrição de uma mudança, quanto uma desmistificação.

Antes do século XVIII, a Filosofia foi o sistema único de organização dos saberes ou, conforme as palavras de Foucault, o "sistema de comunicação dos saberes, uns em relação aos outros"; e nessa direção é que a Filosofia tinha um papel real e operacional no desenvolvimento dos conhecimentos. O projeto da mathesis universali, a procura de um "instrumento formal" que servisse de princípio de legitimação às ciências particulares, preparava de fato a separação entre a ciência - que começaria sua trajetória independente - e a filosofia. Construir-se-ia, agora, sob o horizonte idealizado da ciência unificada, a pluralidade das disciplinas distribuídas em classificações totalizadoras e séries ordenadas. ${ }^{5}$ Conforme $\mathrm{o}$ autor, faz parte desta desmistificação ver, no "século das luzes", não "a luta do conhecimento contra a ignorância, da razão contra as quimeras....", mas "...um imenso e múltiplo combate dos saberes uns contra outros - dos saberes que se opõem entre si por sua morfologia própria, por seus detentores inimigos uns dos outros e por seus efeitos de poder intrínsecos" (FOUCAULT, 1999, p. 214).

\footnotetext{
4 “[...] a tradução sempre leva consigo compromissos que alteram a comunicação. Para fazê-lo necessita saber quais são os aspectos do original que é mais importante preservar e deve também saber algo acerca da formação e experiência daqueles que vão ler seu trabalho. Por que é tão difícil a tradução, seja entre teorias, seja entre linguagens? Porque, como freqüentemente tem sido indicado, as linguagens selecionam o mundo de maneiras diferentes e não dispomos de nenhum meio sub-linguístico neutral de informar." (KUHN, 1975).

5 "Aparece agora, com o disciplinamento dos saberes, em sua singularidade polimorfa, ao mesmo tempo o fato e a regra que agora estão incorporados na nossa cultura e que se chama "ciência". [...] A ciência, como domínio geral, como policiamento disciplinar dos saberes, tomou o lugar tanto da filosofia como da mathesis. E doravante ela vai formular problemas específicos ao policiamento disciplinar dos saberes: problemas de classificação, problemas de hierarquização, problemas de vizinhança, etc.” (FOUCAULT, 1999, p. 218).
} 
O novo entendimento da produção dos conhecimentos científicos como práticas sociais heterogêneas e a valorização do conhecimento incorporado ou habitus facilitam o desenvolvimento dos estudos antropológicos da ciência, entendida como cultura. Para Geertz (1998), considerando a duração temporal das práticas compartilhadas pelos pesquisadores no processo de formação e no desempenho de suas tarefas, ficariam constituídas quase "subculturas" ou comunidades biográficas, versão empírica dos conceitos de Wittgenstein, de "formas de vida" ou "jogos de linguagem".

Nessa direção Collins (1998) estabelece uma equivalência entre as "culturas de evidência" dos cientistas e as "sanções coletivas" comunitárias: além de funcionar como instâncias de arbitragem, pareceriam oferecer uma mediação entre os julgamentos de validade de cada pesquisador, os critérios de julgamento de seus pares e as "epistemologias institucionais" das agências de financiamento e avaliação (GONZALEZ DE GÓMEZ, 2000).

Tais "sujeitos coletivos" da pesquisa constituiriam comunidades de interlocução relativamente homogêneas, com suas próprias linguagens e terminologias especializadas.

Consideradas a pluralidade e a heterogeneidade das práticas de pesquisa e de suas "subculturas" avaliativas, ficariam logo em evidência os problemas que surgem nos contextos da avaliação comparativa (conselhos nacionais de pesquisa, universidades), onde a definição das prioridades e a atribuição de excelência perpassam desde as grandes disciplinas e suas especialidades às áreas inter e transdisciplinares, as quais se configuram hoje não como soma e reunião integradora, mas como uma pluralidade de configurações geradas em torno de diversos parâmetros intertemáticos.

Nesse contexto, a pressuposição de critérios de "certificação universal" só poderia expressar juízos preferenciais de excelência. Isso geraria uma tensão entre a regra apresentada como única e a polinomia das práticas científicas, polinomia que existiria não só entre especialidades ou disciplinas, mas também no interior de uma mesma área, tal como entre a física teórica e a experimental.

Essa tensão seria agravada, aliás, na medida em que fossem menos explicitadas e esclarecidas as diferentes "culturas de evidência" que intervêm como premissas tácitas da autoridade científica na resolução de controvérsias e conflitos (FULLER, 1988). Daí que a reflexividade acerca dessas premissas metacientíficas e tácitas das comunidades envolvidas na produção do conhecimento científico, e sua elucidação em contextos argumentativos, poderia ser um caminho a ser explorado pela avaliação comparativa e interdisciplinar. Para Fuller (1988), existiria em toda prática científica uma "metaciência" implícita no formato de argumentação da disciplina, que intervém como premissa tácita da autoridade científica na resolução de controvérsias e conflitos.

Knorr Cettina (1999) propõe o conceito de cultura epistêmica, ${ }^{6}$ para substituir o de disciplina ou especialidade e dar conta das mudanças próprias da transição a uma sociedade do conhecimento. Ela não só reuniria diferentes especialistas e conhecimentos científicos, mas seria a expressão da inserção da "máquina de conhecimento" além dos espaços acadêmicos ou da esfera produtiva, também nos contextos cotidianos e locais de ação. ${ }^{7}$

\footnotetext{
6 " $[\ldots]$ in replacing notions such as disciplines or specialty with that of an epistemic culture, I want to amplify the knowledge machineries of contemporary sciences until they display the smear of technical, social, simbolic dimensions of intricate experts systems. [...]"Magnifying this aspect of science -not its production of knowledge but its epistemic machinery- reveals the fragmentation of contemporary science; it displays different architectures of empirical approaches, specific constructions of the referent, particular ontologies of instruments, and different social machines. In other words, it brings out the diversity of epistemic cultures. This disunifies the sciences; it runs counter to an assumption generally associated with the work of the Vienna Circle of more than fifty years ago, particularly the argument of the unity of science. "(KNORR CETINA, 1999).
} 
A heterogeneidade das culturas epistêmicas, de modo de gerar "n" parâmetros de configurações transdisciplinares de conhecimentos, seria uma das afirmações centrais de Knorr Cettina.. Como manifestação cultural, os conhecimentos científicos partilham as características da cultura: sendo um traço permanente de toda a humanidade, ao mesmo tempo não existiria um traço cultural que caracterizaria o homem como ser genérico, em todo espaço e todo tempo de sua realização. ${ }^{8} \mathrm{O}$ conceito de "cultura epistêmica" permitiria a releitura de procedimentos pragmáticos de avaliação. O que enuncia, aliás, as condições de resolução do problema, antes de definir um mecanismo generalizável de avaliação.

\section{Da crise da ciência à ciência estratégica}

A opacificação da ciência se fortalece, além daquelas afirmações acerca da heterogeneidade de suas práticas, pelos novos rumos da pesquisa estratégica.

$\mathrm{Na}$ década de 1930, encontramos várias indicações de desestabilização do estatuto prévio da ciência nos países capitalistas. Enquanto a produção de conhecimento científico ficava cada vez mais sujeita à pressão estrutural das esferas produtivas e do Estado, a ciência instituída foi sendo associada às situações de crise que colocavam em suspenso as certezas racionais do "espírito europeu". Lembramos que Husserl escreveria Die Krisis der europaischen Wissenschaft und die transzendentale Phaenomelogi, em 1936, onde "crise" deveria entender-se, naquele momento, não tanto como dúvida acerca da cientificidade, e mais como redução e perda do sentido que a ciência tem tido e poderia ter para a existência humana (XIGAUD, 1976, p. 247 et seq.)

Nesse contexto, a ciência e o trabalho intelectual serão "tematizados" a partir de diferentes ângulos, mas sempre com destaque para a relação entre os conhecimentos e as esferas sociais de sua destinação.

Pouco antes de sua morte (1937) Gramsci desenvolveu o conceito de intelectual orgânico, incorporado à estrutura de poder frente à qual lhe corresponde assumir um papel políticoideológico de articulador do espaço simbólico da classe social à qual pertence. Seu ponto de vista se confronta com a concepção do intelectual como categoria ocupacional (OIT), de intelligentsia (Mannheim) e de elite (Pareto). ${ }^{9}$

\footnotetext{
7 "If the argument about the expanding presence of expert systems and knowledge processes in contemporary Western Societies is right, however, the design stance defeats the intention of understanding this particular development. Not only does the expansion of expert systems result in a massive encrease in the technological and informational products of knowledge process. It also amplifies the process themselves, and knowledge - related contexts and structures. A knowledge society is not simply a society of more experts, more technological gadgets, more specialist interpretations. It is a society permeated with knowledge cultures, the whole set of structures and mechanisms that serve knowledge and unfold with its articulation. (KNORR CETINA, 1999, p.8).

${ }^{8}$ The concept of culture is ussually used to refer to specific historical forms, such as the state ("national culture"), the economy ("market culture"), or the business firm ("organizational culture"). Cultural specificities arise and thrive, one assumes, when domains of social life become separated from one another -when they "curl up upon themselves"- for a period of time. In other words, they thrive in internally referential systems - for example, domains in which participants orient themselves more toward one another and previous system-states than toward the outside. Science and expert systems are obvious candidates for cultural divisions; they are pursued by groupings of specialists who are separated from other experts by institutional boundaries deeply entrenched in all levels of education, in most research organizations, in career choices, in our general system of classification" (KNORR CETINA, 1999, p.2).

${ }^{9}$ Ver BUCI-GLUKSMANN (1980). Ver também as seguintes referências bibliográficas, confirmatórias de nossas anotações: MANNHEIM (1985); MANNHEIM (1993).
} 
A importância da relação entre o conhecimento e sua destinação social foi colocada a par das relações dos conhecimentos entre si, em processos de divisão e reunião que, sem os freios da tradição, os diversificavam e modificavam em ritmo acelerado. Sem falar de interdisciplinaridade, Ranganathan (1932), na esfera dos estudos biblioteconômicos e da documentação, dedicou aos processos de formação e transformação dos conhecimentos uma parte significativa de seus estudos sobre a Teoria das Classificações. ${ }^{10}$ Preocupou-se também com a apropriação dos conhecimentos modernos e locais por aquelas categorias sociais mais sujeitas à exclusão: as mulheres, as crianças, os camponeses - numa Índia em processo de reconstrução pós-colonial.

Após a Segunda Guerra Mundial, existiu a vontade de incorporar tecnologias e descobertas da pesquisa desenvolvidas na fase bélica, nas esferas do Estado, da economia e da sociedade civil.

$\mathrm{Na}$ versão liberal, era possível harmonizar as duas vocações numa política responsável e numa ciência livre. Em 1945, Bush, engenheiro do MIT e então diretor da Office of Scientific Research and Development, do governo federal, com a memória do sucesso do programa nuclear, que tivera o forte suporte da física teórica e experimental, afirmaria que "[...] o livre jogo dos intelectos livres, trabalhando em assuntos de sua própria escolha, na maneira que lhes for ditado pela sua curiosidade para explorar o desconhecido" (BUSH, 1945, p.17).

Era responsabilidade do Estado manter o fluxo de recursos com a mesma abundância que durante a guerra, preservando porém a ciência sua independência para definir agendas de pesquisa e estabelecer prioridades no investimento dos recursos. ${ }^{11}$

Uma idéia de Bush que ficaria registrada na história da política científica seria assim essa afirmação do valor da pesquisa básica e de sua necessidade para o desenvolvimento tecnológico e industrial de um país. Em sua versão, de certo nominalismo, as realizações individuais dos cientistas, trabalhando no desenvolvimento singularizado de disciplinas altamente especializadas, redundaria numa ciência progressiva e benéfica para as sociedades que as patrocinam. A convergência espontânea das plurais ofertas científicas e a demanda social pareceria pressupor a intervenção daquela "mão invisível", que Polanyi já tinha transladado da economia de mercado para a esfera da produção científica. Ou, finalmente, os problemas de vinculação ficariam resolvidos, pela utilização dos meios. Sustentava-se assim uma visão da ciência onde suas manifestações singulares, hegemonizadas pelas ciências hard e lideradas pela física, seriam unificadas por seus efeitos alinhados em direção a um progresso contínuo.

Essa manifestação de otimismo racionalista seria rapidamente interceptada por outras vozes.

$\mathrm{Na}$ década de 1960, predominaria a desconfiança naquela harmonia espontânea entre o desenvolvimento das ciências e os objetivos econômicos e políticos, mas isto levaria a aumentar e a explicitar a aposta na mediação tecnológica.

Ao mesmo tempo em que se intensificam as relações do Estado e da indústria com a ciência (o que na América Latina acontecera sob as doutrinas de segurança e desenvolvimento), foi

\footnotetext{
${ }^{10}$ Conforme BEGTHOL (2004): "Modern classification researchers and classificationists have suggested various terms for the ways in which disciplines can be combined and connected.... Earlier, S. R. Ranganathan had enumerated eleven methods that could be used to combine subjects: loose assemblage (two types); lamination (two types); fission; dissection; denudation; fusion; distillation; clustering/subject bundle; and agglomeration/partial comprehension"

11 A publicação do relatório de Vannevar Bush, Science: the endless frontier, em 1945 (ver BUSH, 1945), teria influenciado para que, em 1950, fosse criada a National Science Foundation (NSF), agência que assumiria a oferta de estímulo e recursos à pesquisa básica
} 
intensificado o processo de opacificação da ciência. Já nos referimos em outra oportunidade a alguns dos discursos críticos provenientes da mesma academia ${ }^{12}$.

Não seria alheia a esta nova crise, porém, a consolidação da pesquisa estratégica, colocando em suspenso alguns dos atributos que eram definidores da ciência: o seu caráter público e a valorização da crítica por pares como condição epistemológica e metodológica de sua realização. Além da restrição à circulação de informações, a tomada de decisão acerca da pesquisa seria em princípio diferenciada e externa à tomada de decisão dos próprios cientistas. A pesquisa estratégica ou orientada por missão teria sua finalidade e sua direção pré-estabelecidas antes de toda escolha - epistêmica, temática, metodológica - dos pesquisadores.

Os termos big science e "ciência orientada por missão" designam uma vinculação pragmática de especialistas, gestores, agentes do Estado e das indústrias, reunindo sob uma mesma meta conhecimentos e recursos, como nos grandes programas de pesquisa que caracterizaram a "conquista espacial".

As formas de vincular conhecimentos ficariam agora sujeitas às condições da mudança de escala e da pesquisa induzida. Os conceitos de "inteligência científica" e "política científica" preparavam, a nosso ver, a passagem de políticas reguladoras implícitas nas arquiteturas disciplinares a formas exteriorizadas de controle e administração da ciência e da tecnologia.

Por outro lado, o desenvolvimento da pesquisa conforme as estruturas disciplinares e as especialidades não atendia à demanda não-disciplinar da pesquisa estratégica e dos programas orientados à missão. A informação seria olhada naquele momento como instrumento de vinculação.

O controle exteriorizado da produção de conhecimentos científicos crescia de fato através do planejamento, monitoramento e avaliação dos seus empreendimentos.

Nesse contexto, podem distinguir-se dois processos complementares. Um deles, a gestão da ciência, consolida um plano de observação e de "segundo grau" que tem como objeto a própria ciência - mas num olhar que transcende e independe da consciência de seus produtores e requer mecanismos exteriorizados de mapeamento e monitoramento de sua produtividade. Num processo indiretamente complementar, os estudos sociais da ciência, à diferença das epistemologias racionalistas, desenvolverão metodologias de cunho 'externalista', "observacionais" e quantitativas, que permitirão estabelecer e operacionalizar indicadores mensuráveis da produção científica.

As ações de informação, que indexam as ações de pesquisa e seus produtos e marcam sua posição nos planos reguladores da produção científica e da administração de ciência e tecnologia, passariam a integrar o domínio das ações política e administrativamente significativas e, em conseqüência, é a partir desse momento que a política e a gestão da

\footnotetext{
12 "Um outro momento significativo aconteceria na década de 1960, quando era visível e intensa a revisão do contrato da ciência com o Estado e a sociedade. De maneira desordenada, poderíamos lembrar a respeito desde os críticos da Escola de Frankfurt - em especial Marcuse e seu One dimensional man - aos novos estudos da história e sociologia da ciência, entre eles os dos chamados pós-empiricistas, com nomes da importância de Kuhn, Lakatos, Feyerabend, Toulmin, Polanyi. Pode-se dizer que encontros, números temáticos de periódicos reconhecidos, como o Minerva, assim como relatórios oficiais dos governos mais envolvidos com os programas de ciência e tecnologia testemunhavam o alcance e a relevância da questão. É nesse período que fica estabelecida uma relação íntima e problemática entre a inovação no domínio das atividades e tecnologias de informação e o desenvolvimento científico-tecnológico, formulando-se um modelo de políticas de informação científico-tecnológica, já clássico nos estudos da ciência da informação" (GONZALEZ DE GOMEZ, 2002).
} 
informação formarão parte do mesmo plano decisório e prospectivo ao qual pertencem a política e a gestão da ciência e da tecnologia.

\section{A interdisciplinaridade: crise da razão "arbórea", crise da universidade}

É no início da década de 1970 que o discurso da interdisciplinaridade ganha o espaço discursivo da academia e das grandes organizações internacionais, como a Organização para Cooperação e Desenvolvimento Econômico (OCDE) e a UNESCO. Se, na década de 1960, a pesquisa orientada por missão surge à luz dos grandes programas científico-tecnológicos, como os espaciais, tendo como carro-chefe as ciências físicas, a interdisciplinaridade se apresenta como forma de superação da especialização excessiva e como alternativa capaz de melhorar as relações - tidas como distantes - entre a universidade e a sociedade, incluídos maiores aportes da educação superior ao crescimento econômico.

Em 1969, realiza-se em Nice, na França, promovida pela OCDE, a First International Conference on Interdisciplinarity. A mesma tem como tema principal o papel da interdisciplinaridade na universidade moderna, dando ênfase ao caráter interdisciplinar das ciências sociais. ${ }^{13}$

Tratava-se de um discurso epistemológico, de cunho "internalista", preocupado em definir e categorizar as modalidades de vinculação entre os conhecimentos, de maior ou menor grau de complementaridade frente a um maior ou menor grau de segmentação, sobre o pano de fundo de uma universalidade da ciência - irrealizada mas desejável.

Alguns autores associam a emergência dos discursos sobre a interdisciplinaridade, na década de 1970, mais especificamente a uma crise pela que passaria a instituição universitária e suas formas de organizar e coordenar os conhecimentos. A crise epistemológica irromperia à luz de uma crise política e social. Conforme Follari (1995), a tematização da interdisciplinaridade estaria relacionada nesse momento a uma crise de legitimação dos regimes capitalistas constituídos, que teria sua expressão nos movimentos estudantis em Paris, em Berkeley, assim como nas universidades latino-americanas. ${ }^{14}$ De fato, Bourdieu, no seu livro Homo Academicus (1984), em especial no capítulo 5, analisa os acontecimentos de maio de 1968 como efeito de uma perda relativa de crédito dos diplomas e das posições profissionais dos setores menos favorecidos dentro das populações universitárias. Bourdieu, nesse quadro, destaca a união de atores provenientes de diferentes campos científicos e a suspensão provisória dos mecanismos de manutenção da autonomia, permitindo que todos jogassem um mesmo jogo, a despeito das rixas e concorrências intercampos. ${ }^{15}$ Seria um efeito de sincronização entre atores com posições

\footnotetext{
${ }^{13}$ Entre os nomes que se destacaram nessa reunião estão Jean Piaget, Andre Lichnerowicz, Erich Jantsch. Idéias e tendências dessa conferência e dos textos publicados em seus anais, tiveram influência em pensadores brasileiros, como Japiassu (1976) e Fazenda (1991). Em princípio, Jantsch e Bianchetti (1997) identificam na Conferência de Nice uma abordagem da interdisciplinaridade a partir de uma filosofia do sujeito, que veria na fragmentação dos mesmos uma patologia. Em 1982, a mesma OCDE gera um novo estudo relacionando interdisciplinaridade e sociedade, com mais ênfase nas ciências sociais: The university and the community: the problems of changing relationships.

${ }^{14}$ Lembramos que, ao mesmo tempo, em Berkeley e em Paris entraram em confronto Heráclito contra Parmênides e em Buenos Aires, Rosário e outras universidades da América do Sul, se discutia o distanciamento do ensino da realidade latinoamericana.

15 “...l'unification des differents champs qui resulte de la mise en suspens provisoire des mechanismes tendant à maintenir l'autonomie relative de chacun d'eux, engage dans le même jeu, avec des positions identiques, des agents occupant jusque-là dês positions homologues dans des champs différents.” (BOURDIEU, 1984, p. 228).
} 
equivalentes em seus respectivos campos disciplinares, o que permitiria a desativação relativa e temporária do princípio de autonomia de cada campo ou subcampo singular. A vinculação assim estabelecida seria porém de natureza política e não incluiria, para o autor, considerações específicas acerca dos conteúdos e das práticas disciplinares. Pela mesma sincronia, a crise, sendo de início universitária, tinha condições "estruturais" de generalização. O vínculo político era extensível a determinados setores dos trabalhadores e seus coletivos organizados.

Sem ignorar as abordagens críticas, seria interessante colocar aquela "problematização" própria dos discursos sobre a inter- e a transdisciplinaridade, visando superar as lógicas "arbóreas" instituídas nos conhecimentos formalizados, junto a outros discursos igualmente datados na década de 1970. Esse terceiro grupo teria em comum destacar o papel dos conhecimentos na economia e nos processos de trabalho, muitas vezes associado a uma visão prospectiva da sociedade, a ser chamada "sociedade do conhecimento" ou "sociedade da informação".

Para citar alguns dos mais significativos, Braverman publica em 1974 Labor and monopoly capital: the degradation of work in the Twentieth Century e Daniel Bell publica em 1973 The coming of post-industrial society, onde apresenta seus conceitos de informação como recurso e do conhecimento como commodity.

\section{A transdisciplinaridade: novo regime de conhecimento}

Na década de 1990, seria a vez de pensar a transdisciplinaridade, associando novas demandas éticas e políticas à busca de inovações epistemológicas.

Uma das expressões mais importantes dessas tendências pareceria ser o Premier Congrès Mondial de la Transdisciplinarité, realizado no Convento da Arrábida, em Portugal, em 1994, promovido por um Grupo de Estudo da Transdisciplinaridade da UNESCO. Contou com mentores como Basarab Nicolescu e Edgar Morin, enunciando como pilar do projeto transdisciplinar o princípio da imprevisibilidade, propulsor do pensamento da complexidade. ${ }^{16}$ Outro evento, o Congresso Internacional de Locarno, realizado na Suíça, entre 30 de abril e 2 de maio de 1997, foi dedicado a pensar a transdisciplinaridade na universidade.

A questão da transdisciplinaridade aparece agora no contexto de discursos engajados em questões ético-politicas: do compromisso ecológico com o desenvolvimento sustentável, a "epistemologia feminista", o questionamento do papel dos especialistas nas cadeias decisórias e a participação da sociedade civil e dos atores locais na definição de problemas e agendas de pesquisa, inclusão presente na expressão de "ciência ao ar livre". Um outro núcleo temático se constitui em torno do conceito de colaboração (DOGSON, 2001; SCHUMMER, 2001; MUTSCHKE; HAASE, 2001; MOODY, 2004). No Brasil, entre outros estudos, Léa Velho (1997) apresenta a síntese de um projeto promovido pelo Ministério da Ciência e Tecnologia

\footnotetext{
${ }^{16}$ Entre os organizadores do Congresso do Convento da Arrábida, Portugal, 2-6 novembre 1994, temos a Unesco, com forte participação da Comissão Nacional de Portugal e o Centre International de Recherches et d'Etudes Transdisciplinaires (Ciret), em Paris, o Conselho Europeu, entre outros. Havia representantes de Argentina, Brasil, Austrália, além dos principais países da Europa. No Brasil, o Centro de Educação Transdisciplinar (Cetrans) e o projeto Escola do Futuro, na Universidade de São Paulo ( $<\mathrm{http}$ ://www.cetrans.futuro.usp.br/>), filiam-se às orientações daquele projeto. O Congresso de Arrábida teve outros participantes significativos como Gibbons, Klein, assim como representantes do Brasil e da América Latina. O núcleo das principais idéias e orientações que inspiraram o Congresso seria mantido no CIRET (http://perso.club-internet.fr/nicol/ciret).
} 
(MCT) e a Organização dos Estados Americanos (OEA), sobre a "Cooperação em Ciência e Tecnologia no Mercosul”, que abrange Brasil, Argentina, Uruguai, Paraguai, Chile.

Uma espécie de "plataforma" dos programas transdisciplinares foi o texto de convocação para a International Transdisciplinarity Conference, realizada no Swiss Federal Institute of Technology, Zurich (2000), da qual participaram entre outros, Nowotny e Gibbons, o porta-voz do Modo de Conhecimento $2:{ }^{17}$

- A pesquisa transdisciplinar é baseada em processo [...] Uma razão para tal é que este processo representa também um processo de mediação em que são negociadas as posições de valor e os conflitos de interesses.

c Diferentemente da consultoria, a pesquisa transdisciplinar requer um conhecimento científico que vai além do caso individual.

c A pesquisa transdisciplinar envolve especificamente cooperação entre as ciências sociais, naturais, engenharias e as artes.

- A pesquisa transdisciplinar visa alcançar inovações nas seguintes áreas:

- Sistematização de conhecimento independente das disciplinas assim como capacidade de generalização e de elaboração teórica baseada em conhecimento contextualizado.

- Abordagem na complexidade. [...] sem ajustar-se aos requisitos gnosiológicos de uma disciplina ou de um pesquisador [específicos] $[\ldots]$

- O pesquisador é parte do contexto da pesquisa e tem uma influência sobre o objeto da pesquisa, isto é, sobre as soluções que são reconhecidas e adotadas na sociedade. Nos projetos transdisciplinares, a complexidade inclui o problema de reflexividade e da auto-referência da pesquisa socialmente relevante.

- Inclusão da incerteza e da ignorância nas soluções de problemas. Todo conhecimento científico é de caráter temporário...

- Envolvimento dos atores e do conhecimento local. O modo de olhar a participação nos projetos de transdisciplinaridade remete a uma troca interativa de conhecimento e valores e de mútua aprendizagem. $\mathrm{O}$ conhecimento local [...] referente ao contexto pode ser caracterizado por uma preocupação intensiva e durante longos anos com um objeto de pesquisa, que os cientistas provavelmente teriam dificuldades em obter. O conhecimento local, além disso, é qualitativo e não quantificável e só será formalizado em textos numa extensão limitada. $[\ldots]^{18}$ (KÜFFER, 2000, p.3)"

\footnotetext{
17 O texto de convocação para a International Transdisciplinarity 2000 Conference (Swiss Federal Institute of Technology, Zurique, de 27 de fevereiro a $1^{\circ}$ de março de 2000) - Transdisciplinarity: joint problem-solving among science, technology and society - foi apresentado com a assinatura de Christoph Küffer. A publicação dos anais deu lugar a um livro que tem como editores os mentores da Conferência: Julie Thompson Klein, Walter GrossenbacherMansuy, Rudolf Häberli, Alain Bill, Roland W. Schloz, Myrtha Welti. Ver nas referências KLEIN, J. T. et al.
} 
As demandas de transgressão e dissenso ainda seriam ouvidas na pluralidade de suas vozes. Por um lado, as leituras ditas pós-estruturalistas (Deleuze, Derrida, Lyotard), colocam em questão a esterilização da racionalidade pelas lógicas monovalentes e hierárquicas tidas como compulsórias; por outro lado, os porta-vozes do "Modo 2" do conhecimento sinalizam o caminho da inovação como alternativa para uma versão da ciência isolada em claustros acadêmicos, com risco de equacionar a pesquisa básica com a estagnação da pesquisa. Todas estas vozes seriam logo interceptadas pelas novas tecnologias que têm alterado desde as experiências de laboratório à comunicação formal e informal dos cientistas. Ficaria assim pouco espaço para imaginar, à luz da razão fragilizada, a delicadeza dos vínculos possíveis.

\section{Contrapontos: entre a razão mediada e a razão reflexiva, a razão leve}

18 "[...] Transdisciplinary research is process-based. This process character refers to the basic assumptions, on which the project builds. Insights accrue in the course of an interactive, communicative and recursive research process. One reason for this is that this process also represents a mediation process in which value positions and conflicts of interest are negotiated.

- Unlike consultancy, transdisciplinary research has a scientific knowledge requirement which goes beyond the individual case.

- Transdisciplinary research specifically involves co-operation between the natural, engineering and social sciences and the arts.

- $\quad$ Transdisciplinary research aims to achieve innovations in the following areas:

- Discipline-independent systematisation of knowledge as well as generalisation capacity and theory formation based on context-related knowledge.

- Approach to complexity. The complexity of the problem formulation is dictated by the academic or extra-disciplinary problem framing and it is not tailored to the discipline's or researcher's knowledge requirement. Instead of a discipline-oriented reduction in complexity, what must be developed is a target and action-oriented complexity reduction.

- The researcher is part of the research context and has an influence on the object of research, i.e. the solutions which are considered and adopted in society. In transdisciplinary projects, complexity includes the problem of reflexivity and the self-reference of socially relevant research.

$\circ \quad$ Approach to uncertainty and nescience in problem solutions. Scientific knowledge is temporary in nature. New insights which often emerge in the application phase contest previous knowledge. Urgent problem solutions require methods for the identification of optimum action strategies despite lack of knowledge.

- Transparent negotiation of values and standards which are inseparably linked with the problems.

- Involvement of actors and local knowledge. The vision of participation in transdisciplinary projects refers to an interactive exchange of knowledge and values and to mutual learning. Local knowledge refers to forms of knowledge which are the outcome of a direct reference to the research object. Local context-related knowledge can be characterised by many years of intensive preoccupation with a research object scientists are hardly likely to achieve. Local knowledge is often qualitative and not quantifiable. Local knowledge is often only implicit and the extent to which it can be explicitly formulated in the form of a text is limited" (KUEFFER, 2000, p.3). 
Entre as diversas afirmações acerca da crescente relevância das relações entre conhecimentos, cabe perguntar se estamos ou não frente a uma mudança radical, paradigmática, ou somente num estado de transição, numa modalidade ou etapa dos regimes de conhecimento já instituídos.

Rammert (2004) considera que, para falar de mudança, deveriam encontrar-se evidências de novos padrões de coordenação nos e entre os campos de produção de conhecimentos - o acadêmico, o econômico e o político -, da condensação desses padrões num conjunto coerente de regras de jogo e da substituição dos modos prévios de jogo pelas novas regras. Rammert, numa apresentação simplificada de sua proposta, considera que as sociedades ditas modernas caracterizaram-se por processos de diferenciação funcional, formando-se assim subsistemas com diversas "funções", mas todos eles com os mesmos níveis de estratificação e a mesma relevância. No mundo contemporâneo, processos sucessivos e incrementais de especialização e segmentação teriam levado a uma "quebra" dessas unidades funcionais integradas ou subsistemas, dando lugar a um novo mecanismo, de diferenciação fragmentada, onde os conhecimentos se produzem em redes heterogêneas e de forma distribuída - de modo que o cumprimento de objetivos comuns não implicaria tempos e espaços homogêneos de compartilhamento das tarefas. Grupos heterogêneos de peritos formariam comunidades de práticas não disciplinares, construiriam um patchwork de diversas disciplinas. Existiria lugar, neste novo regime de conhecimento, para redes "híbridas", que não se apóiam nas relações contratuais de mercado nem nas relações funcionalmente definidas por organizações, e nas quais têm lugar importante as relações informais e de confiança.

As tecnologias terão um papel da maior importância nessa reformulação do regime dos conhecimentos, mas dentro de um novo estilo gnosiológico.

Entre os estilos de conhecimento da modernidade, um era bastante bem estabelecido, caracterizando-se por dois movimentos complementares: a) a passagem dos conhecimentos concretos, contextuais, situados, aos conhecimentos abstratos, formalizados, relativamente livres de contexto; b) a passagem dos conhecimentos incorporados, tácitos, implícitos, aos conhecimentos explicitados, codificados, inscritos.

Com o advento das tecnologias digitais, interativas e da hipermídia, cresceu num primeiro momento a demanda por explicitação, formalização e codificação dos conhecimentos, em inscrições padronizadas. Quanto maior grau de formalização, mais próximo estaria o conhecimento de um estado de finalização para, então, prestar-se a diversas e indefinidas formas de utilização.

A informação, entendida como manifestação digital e controlável de valores semânticos, em si mesmos imprevisíveis, tem sido uma instância decisiva nesses processos de explicitação.

Para Rammert (2004), no novo regime de conhecimento, estaria produzindo-se uma mudança de efeitos radicais. Em sociedades globalizadas e sob condições definidas por referência às políticas de inovação, os conhecimentos se mobilizariam, ao mesmo tempo, em direção a uma maior incorporação de tecnologias ${ }^{19}$ e de uma redução das exigências e operações de codificação e explicitação. ${ }^{20}$ A explicitação, cada vez mais custosa, daria lugar ao aumento do papel do

\footnotetext{
${ }^{19}$ Tal seria o caso das tecnologias genéricas: "The spectrometer, the computer-simulation, the electronic microscope are examples of this type of generic technologies, around which particular communities of knowledge came up [...]. These communities circulated both, explicit and codified knowledge, via special journals and tacit knowing via technical experience" (RAMMERT, 2004, p.18).

20 "If we accept the description of the actual knowledge changes towards a more heterogeneous and distributed knowledge production, and if we acknowledge the increasing use of a growing variety of technical instruments, then we may discover another style of knowing. It is more sensible for the relations between explicit and non-explicit
} 
conhecimento tácito e incorporado, estabelecendo-se novos modos de interação com o formal e codificado. Isto se deveria não só a cada vez maior integração da pesquisa com tecnologias, instrumentos e máquinas - o que sempre requer um conhecer não verbalizado - mas também ao fato de que, com a fragmentação dos diferentes domínios de conhecimentos, diminui cada vez mais a parte da população que pode lidar de modo explícito e competente com todos os códigos e manuais requeridos para interpretar devidamente os meios de uma ação científico-tecnológica complexa (RAMMERT, 2004, p.15).

Poderíamos observar, como uma primeira restrição, que diferentes "culturas epistêmicas" (KNORR CETTINA, 1999) terão diferente modo de relação com as tecnologias ditas genéricas e as infra-estruturas analógico-digitais.

Enquanto, na análise de Rammert, existiria uma tendência à redução das interações mediadas pela linguagem a par do fortalecimento das mediações tecnológicas, Habermas faz uma aposta alternativa na razão plural e reflexiva. Faz um resgate do argumento da Escola de Frankfurt acerca do papel emancipatório da reflexividade, entendida por ele como processo discursivo e intersubjetivo, e não como exercício introspectivo da consciência. Para Lucia Aragão existiria um motivo importante, além dos teóricos e metodológicos, para escolher a linguagem ${ }^{21}$ como a face do humano em que se revela a razão: " $\mathrm{E}$ o fato da linguagem ter por propósito próprio fomentar o entendimento entre os homens, e não a dominação" (ARAGÃO, 2002, p. 108).

Os sujeitos capazes de fala e ação podem ter frente à linguagem uma atitude dependente ou autônoma, assim como se defrontarem com diferentes modalidades de racionalização societária.

Habermas considera, assim, duas categorias de racionalização societária, ${ }^{22}$ relacionadas a dois modos diferenciados de integração social. O que faz a diferença entre os dois modos de integração seria o fato da coordenação da ação passar ou não pela consciência dos indivíduos em sua capacidade de agente e, em conseqüência, incluindo ou não nas tomadas de decisão o ponto de vista dos participantes: a integração funcional, própria do sistema; a integração social, própria do "mundo da vida", que acontece no domínio da reprodução cultural, socialização e integração social. Apenas mecanismos sociais de integração, mediados pelo uso da linguagem, e não funcionais, mediados por meios de controle (steering media), permitem uma ordem social intersubjetivamente compartilhada, possibilitando a construção e a estabilização de redes

aspects of knowledge, but it does not rely on the fundamental and formal processes of explicitation . It produces coherence between heterogeneous participants by processes of enculturation that constitute communities of knowing by interaction, learning and soft theorizing". (RAMMERT, 2004, p.18).

${ }^{21}$ Seria interessante lembrar que o caminho de Habermas, entre a filosofia e a sociologia, foi de "reconstrução". As expressões e fórmulas proposicionais da gramática e da lógica nos forneceriam uma área de objetos formais acessíveis publicamente, ainda que restritos, em sua versão inicial, a análise lógico-semântica, prescindindo da situação de fala, do uso da linguagem, seus contextos, das demandas e "papéis dialogais" dos falantes, ou seja, das considerações pragmáticas. A reconstrução das regras pressupostas nos usos quotidianos da linguagem, permitiriam quebrar os limites de uma filosofia da ciência, driblando tanto o logocentrismo como o contextualismo (HABERMAS, 1985, v.1, p. 318; HABERMAS, 1985, v. 2, p. 400). Com efeito, ao mesmo tempo em que "publicitadas" pela linguagem e abertas à crítica, o que faz com que esses objetos formais ou condições estruturais da comunicação se projetem além das situações imediatas e locais de sua manifestação, elas só se conhecem e manifestam num uso local da linguagem, o que mantém sua reconstrução dentro das perspectivas "falibilistas" de toda empiria. Habermas denomina a essa reconstrução que mantém relações de suporte e realimentação com a Teoria da ação comunicativa, uma "pragmática formal” ou "pragmática universal" (HABERMAS, 1990, p. 48 et seq.).

${ }^{22}$ Para compreender o que seja racionalização, devemos afastar um primeiro significado atribuível à expressão, de disfarçar e ocultar motivos subjacentes e intenções; para Habermas designa uma lógica de coordenação da ação coletiva. 
regulares de interações e deixando em aberto a possibilidade de processos argumentativos e heurísticos, de crítica e aprendizagem social.

"Mundo de vida" designaria as esferas da sociedade simbólica e comunicacionalmente estruturadas; o estoque de saberes implícitos e intuitivos e as práticas socialmente estabelecidas que servem de contexto a todo entendimento; e funcionaria ao mesmo tempo como restrição e como recurso. (HABERMAS, 1984, v. 1, p. 70). A relação entre mundo de vida e a ação comunicativa é dialética: se o mundo de vida é condição "possibilitadora" da ação comunicativa, ele é reproduzido pelos mecanismos integrativos da ação comunicativa.

As condições do uso da linguagem quotidiana possuiriam um "potencial de racionalidade", a partir de onde constrói seu conceito de "razão comunicativa".

Ação comunicativa é uma forma de interação social na qual o plano de ação de vários agentes é coordenado mediante o intercâmbio de atos comunicativos - através do uso da linguagem verbalizada ou de expressões extra-verbais correspondentes - orientadas à obtenção do entendimento (Verstandingung). ${ }^{23} \mathrm{Na}$ ação comunicativa, a racionalidade constitui só um potencial crítico; a racionalidade comunicativa é a expressão desse potencial.

Se a teoria da ação comunicativa remete às sociedades modernas, a racionalidade comunicativa faz sentido em sociedades modernas que têm desenvolvido formas pós-convencionais de ação coletiva. Existiria uma distinção importante, entre ação de comunicação convencional e ação de comunicação pós-convencional; ${ }^{24}$ só esta segunda forma coincidiria com as formas de argumentação críticas e de "final-aberto".

Com efeito, poderíamos diferenciar três níveis de reflexividade em Habermas - argumento adaptado do texto de Maeve Cooke (1997). A relação reflexiva com o mundo se estabelece, em primeiro lugar, quando os participantes num ato de comunicação colocam demandas de validade que podem ser reciprocamente aceitas ou negadas. De fato, a habilidade para adotar diferentes atitudes em relação aos mundos correspondentes seria a precondição para uma relação reflexiva com o mundo (HABERMAS, 1984, v. 1, p. 66).

Habermas considera essa relação reflexiva com o mundo como uma relação indireta, já que é mediada pela necessidade de reconhecimento intersubjetivo das demandas de validade. Daí que os participantes da ação comunicativa só podem alcançar seus objetivos cooperativamente. Os meios de sucesso não estão disponíveis para um agente individual; cada um depende da cooperação e de reconhecimento dos outros.

Para Habermas, compreendemos um ato discursivo quando compreendemos o que o faz aceitável. Para a semântica da verdade, o entendimento da sentença e o entendimento da verdade da sentença não podem ser separados da relação inerente da linguagem com a validade dos

\footnotetext{
${ }^{23}$ As teses mais fortes estariam na consideração do consenso como o mecanismo fundamental na coordenação social no mundo de vida. O termo Verstandingung porém seria de difícil tradução. Cooke observa que a translação em inglês como understanding se apóia no duplo significado do termo nessa língua inglês, "compreensão" e "acordo" [agreement] - ainda que neste segundo sentido pouco usado na língua comum inglesa: "For Habermas, "consensus" as a mechanism of social coordination is connected with the idea of Verstandigung. A notoriously difficult word to translate, Verstandingung refers both to linguistic understanding and to the process of reaching agreement, thus extending across a spectrum of meanings ranging from comprehension to consensus." (COOKE, 1997, p. 9).

24 O conceito, apropriado das teses de Kolberg, designaria "a completa reversibilidade dos pontos de vista a partir dos quais os participantes apresentam seus argumentos: a universalidade, no sentido de uma inclusão de todos os concernidos; finalmente, a reciprocidade do reconhecimento igual das pretensões de cada participante por todos os demais" (HABERMAS, 1989). Para Maeve Cooke, essa diferenciação seria mais importante do que o próprio Habermas pareceria considerar.
} 
enunciados. Assim, conhece-se o significado de uma palavra quando se conhece sua contribuição à "potência de verdade" de uma frase formada com sua ajuda; o significado de uma sentença é determinado por suas condições de verdade. A teoria semântica da verdade limita, porém, suas afirmações às proposições declarativas (afirmativas ou negativas). Um primeiro passo no desenvolvimento da teoria da ação comunicativa será dado por Habermas na direção de incorporar outras demandas de validade. Para isso, é necessário generalizar um conceito de validade que permita ir além da verdade proposicional e que coloca as condições de validade não ao nível da semântica das sentenças, mas ao nível da pragmática da enunciação.

O segundo sentido de reflexividade se estabelece pela diferenciação entre ação comunicativa e argumentação, que só seria possível na sociedade pós-convencional, e através da constituição de uma esfera de crítica intersubjetiva dos critérios formais de validação, e que denomina diskours (discurso). "Discurso" designa assim a forma de comunicação caracterizada pela argumentação, na qual as demandas de validade que se tornarem problemáticas são "tematizadas", "virtualizadas" e sujeitas a exame, para estabelecer se são ou não legítimas. Para iniciar um discurso temos que sair dos contextos da ação e da experiência: nos discursos não intercambiamos informações, mas argumentos que servem para justificar ou rejeitar pretensões de validade problematizadas (HABERMAS, 1994).

Reflexivo, num terceiro sentido, remete à distinção entre a tematização de demandas de validade (validity claims) nas práticas comunicativas da vida cotidiana e o desenvolvimento de formas institucionalizadas e especializadas de argumentação ("culturas de peritos").

Reserva de potenciais de racionalização e aprendizagem coletiva, os processos reflexivos foram se distanciando dos mundos da vida, deixando os processos de legitimação em mãos exclusivas dos especialistas e provocando o "encapsulamento" dos processos de aprendizagem, distanciamento reforçado pela colonização do mundo da vida por processos redutores de racionalização seletiva. ${ }^{25}$

Não existiria justificativa para esses processos dissociativos, senão em projetos de dominação. Para Habermas, a linguagem da política deveria ser o mais próxima possível de uma linguagem comum, já que tanto o leigo como o especialista são igualmente capazes e incapazes de participar da tomada de decisão com o apoio de fóruns de aconselhamento ou consultoria e de diálogo entre saberes, pois não existe disciplina nem profissão capaz de dar conta sozinha dos plurais conhecimentos necessários à elaboração e à implementação de políticas.

Daí que a primeira expectativa de vinculação de conhecimentos, ações e sujeitos estaria nessa superação da colonização do mundo da vida e das formas de coordenação social que privilegiam os meios, sem incorporar saberes e questões de todos os participantes.

Entre outros aportes de Habermas para nossas questões, destacaríamos sua concepção da pluralidade das demandas de validade, vinculadas ao potencial de racionalidade do uso comunicativo da linguagem; além de alargar a racionalidade além de seu uso instrumental e representativo, com desdobramentos numa racionalidade ética e estética, oferece-nos perspectivas para pensar as interações entre a ciência, a arte, a ética, o direito - e outras esferas dissociadas pela modernidade -, de modo que pudessem rebater umas nas outras na reconsideração de seus limites e suas possibilidades. É igualmente interessante, para uma epistemologia não-disciplinar, o distanciamento pragmático que ele introduz entre a meta ilocucionária e o conteúdo locucionário ou proposicional dos atos enunciativos, distanciamento que permitiria as mudanças de perspectivas e o trânsito dentro dos e entre os mundos ou das

\footnotetext{
${ }^{25}$ Isso se manifesta em patologias: perda de sentido (no contexto da reprodução cultural), anomia (no contexto da integração social), desordens psicológicas (no contexto da socialização e formação da identidade).
} 
atitudes com relação ao mundo. De fato, sua abordagem pragmática, ao gerar um medium interpessoal de coordenação da ação e de organização da direção e seqüências da interação, em qualquer cenário de práticas sociais, incluiria também movimentos e cruzamentos indefinidos entre diferentes alternativas epistêmicas, suas normativas e suas práticas.

Se nesta concepção as possibilidades em aberto se defrontam com crises e patologias que as inviabilizam, outras abordagens têm como ponto de partida expectativas por vezes mais modestas, por vezes mais radicais.

Eco contrapõe o pensamento linear, prefigurado no "dicionário" das definições únicas e próprio do pensamento forte, um outro modelo, o da enciclopédia, do pensamento leve, das configurações de labirinto, rizoma, rede. De fato, o projeto enciclopédico, visando reunir e organizar a diversidade dos saberes numa totalidade somatória, partia já do reconhecimento de sua impossibilidade:

[...] O sistema geral das ciências e das artes é uma espécie de labirinto, de caminho tortuoso que o espírito enfrenta sem conhecer bastante a trilha que deve seguir... Essa desordem, assim, ...vai desfigurar, senão anular por completo, a árvore enciclopédica que o quiser representar. (D'ALÉMBERT apud ECO, 1990).

Nos textos provenientes dos estudos da cultura e de um pensamento dito pós-colonial, que inclui autores latino-americanos como Martin Barbeiro e Canclini, entre muitos outros, a questão epistemológica converge na questão cultural de cruzamento das fronteiras - entre conhecimentos, entre países, entre etnias - revigorando o pensamento da "alteridade". Para alguns desses autores, as relações de transversalidade não aconteceriam entre um espaço local e um espaço global, mas sim entre uma teoria e outra teoria, ou entre uma teoria do centro e sua "recodificação" em circunstâncias periféricas (TORO, 2004, p. 291). Um conceito expressivo dessas conjunturas seria o de "hibridez", que designaria uma estratégia em dois momentos: primeiro, trata-se de potencializar a diferença e não de reduzi-la; num segundo momento, partese do reconhecimento das diferenças, estabelecendo-se um processo de negociação de identidades diferentes num terceiro espaço, o in-between de Bhahba (ver entre outros, TORO, 2004, p.304-7).

Klein e Dogan já usaram também o conceito de hibridez na área das ciências para referir-se a formas de relação entre conhecimentos que não se estabelecem entre especialidades ou disciplinas, no todo ou em partes significativas, mas implica zonas de negociação, pontos de cruzamento, articulações sem formalizações, transgressão das demarcações fixas de territórios epistêmicos.

Guattari (1987) opõe a transversalidade aos padrões de relacionamento lineares e hierárquicos, em qualquer de suas manifestações; para Deleuze (1992), trata-se de introduzir o movimento onde o pensamento fica enrijecido na busca de invariâncias - não movimento a partir do ponto de apoio, mas movimento de onda; não a partir da origem, mas no "chegar entre". Trata-se de "rachar" as palavras e as coisas, a invenção de novas sintaxes, novos sentidos, novos agrupamentos que as tecnologias poderiam possibilitar, mas não iniciar. A informação, antes que expressão da novidade, pareceria caracterizar hoje a impossibilidade de anunciar o advento do novo (DELEUZE, 1992). 
Não seria fácil estabelecer as linhagens de família de todas estas abordagens. Caberia interrogar, de novo, o que provoca a busca das linhas de fuga, do transgredir, do "rachar", de buscar fendas; provocação que atenta ora para o pensamento que desliza, ora para o objeto que provoca.

Por um lado, de inúmeras esquinas nos chegam experiências do "rachamento" das categorias identitárias, de comoções da racionalidade que não se aquietam por sua simples negação. Por outro lado, nada indica qual seria a eficácia da razão "moderada" num cenário em que se pretende transferir aos meios a competência dos agrupamentos e das vinculações. Ou quiçá o que desativa as certezas do "pensamento forte" não seja alheio à própria presença e à força da tecnologia que, como dynamis, exibe hoje o que desde sempre a caracterizaria, a geração de uma disponibilidade e de uma potência que não ficam atreladas nem a uma origem nem a uma finalidade, ainda que, no contexto da sociedade produtivista, obtenha um término, como produto (AGAMBEM, 1994, 64-65).

A indagação teria que ganhar uma nova espessura: acerca de como a "vida nua", lugar comum das vivências do híbrido, do transversal, do singular, do movimento, poderá habitar a pólis de uma ciência exposta à radicalidade da pergunta tecnológica.

\section{Referências}

AGAMBEN, G. The man without content. Palo Alto: Stanford University Press, 1999.

AMARAL, M. B. A disciplina da natureza e a natureza das disciplinas: a ciência como produção cultural: relatos de um encontro com Timothy Lenoir. Episteme, Porto Alegre, v. 2, n. 4, p. 11726, 1997.

ARAGON, L. Habermas, filósofo e sociólogo de nosso tempo. Rio de Janeiro: Tempo Brasileiro, 2002.

BEGHTOL, C. Exploring new approaches to the organization of knowledge: the subject classification of James Duff Brown. Library Trends, Spring, 2004.

BELL, D. O advento da sociedade pós-industrial: uma tentativa de previsão social. São Paulo : Cultrix, 1973.

BORDIEU, P. Homo academicus. Paris: Minuit, 1984.

BOWKER, G.; STAR, S. Social theoretical issues in the design of collaboratories: customized software for community support versus large-scale infrastructure. In: OLSON, G. M.; SMITH, J. B.; MALONE, T. W. Coordination theory and collaboration technology. Mahwah, NJ: Lawrence Erlbaum Associates, 2001. Disponível em: $<$ http://weber.ucsd.edu/ gbowker/pubs.htm>. Acesso em: mar. 2005.

BRAVERMAN, H. Trabalho e capital monopolista: a degradação do trabalho no séc. XX. Rio de Janeiro: J. Zahar, 1980

BUCI-GLUCKSMANN, C. Gramsci e o Estado. Rio de Janeiro: Paz e Terra, 1980. 
BUSH, V. Science, the endless frontier: a report to the president by Vannevar Bush, Director of the Office of Scientific Research and Development. Washington: United States Government Printing Office, 1945.

CALLON, M. et al. Réseau et coordination. Paris: Economica, 2003.

CHUBIN, D. E. The conceptualization of scientific specialities. The Sociological Quarterly, n. 17, p. 448-76, 1976.

COLLINS, H. M. The meaning of data: open and closed evidential cultures in the search for gravitational waves. American Journal of Sociology, v. 104, n. 2, p. 293-337,1998.

COOKE, M. Language and reason: a study of Habermas's pragmatics. Cambridge, MA: The MIT Press, 1997. 1. ed. 1994.

DELEUZE, G. Conversações. Rio de Janeiro: Ed. 34, 1992.

DODGSON, M. Strategic Research partnerships: their role, and some issues of measuring their extent and outcomes - experiences from Europe and Asia. In: NATIONAL SCIENCE FOUNDATION WORKSHOP: strategic research partnerships, 2000, Washington. Proceedings...Arlington: National Science Foundation, Division of Science Resource Studies, 2001.

DOGAN, M. The hybridization of social science knowledge. Library Trends, v. 45, n. 2, p.296314, Fall 1996.

ECO, U. El antiporfirio. In: VATTIMO, Gianni; ROVATTI, Pier Aldo (Ed.). El pensamiento débil. Madrid: Catedra, 1990.

FOUCAULT, M. Em defesa da sociedade. São Paulo: Martins Fontes, 1999.

FULLER, S. Social epistemology: science, technology and society. Bloogmington: Indiana University, 1988.

GEERTZ, C. O saber local. Petrópolis: Vozes, 1998.

GONZÁLEZ DE GOMEZ, M. N. Da organização do conhecimento às políticas de informação. Informare, Rio de Janeiro, v. 2, n. 2, p.58-66, 1996. 2000. . O caráter seletivo das ações de informação. Informare, Rio de Janeiro, v. 5, n. 2, p.7-31,

GUATTARI, F. Revolução molecular: pulsações políticas do desejo. 2. ed. São Paulo: Brasiliense, 1987.

HABERMAS, J. Agir comunicativo e razão destranscendentalizada. Rio de Janeiro: Tempo Brasileiro, 2002. . Consciência moral e agir comunicativo. Rio de Janeiro: Tempo Brasileiro, 1989.

Catedra, 1994. . Teoria de la acción comunicativa: complementos y estudios posteriores. Madrid: 
HECKHAUSEN, H. Interdisciplinarity: problems of teaching and research in universities. Nice: CERI, 1970.

JANTSCH, Ari P.; BIANCHETTI, Lucídio. Interdisciplinaridade: para além da filosofia do sujeito. Petrópolis: Vozes, 1997.

KANFER, A.; BRUCE, B. C.; HAYTHORTHWAITE, C; BURBULES, N.; WADE, J.; BOWKER, G.; PORAC, J. Modeling distributed knowledge processes in next generation multidisciplinary alliances. In: ACADEMIA/INDUSTRY WORKING CONFERENCE ON RESEARCH CHALLENGES, 2000, Buffalo. Proceedings...Los Alamitos: IEEE Computer Society, 2000.

KLEIN, J. T. Interdisciplinary needs: the current context. Library Trends, v. 45, n. 2, p.134-53, Fall 1996.

Notes toward a social epistemology of transdisciplinarity. In: CONGRESSO MUNDIAL DA TRANSDISCIPLINARIDADE, 1., 1994. Disponível em: <http://perso.clubinternet.fr/nicol/ciret/bulletin/b12/b12c2.htm>. Acesso em: mar. 2005.

. Unity of knowledge and transdisciplinarity: contexts of definition, theory and the new discourse of problem solving. In: INTERNATIONAL ENCYCLOPEDIA OF LIFE SUPPORT SYSTEMS. Oxford, UK: Eolss Publishers, 2001. Disponível em: <http://www.eolss.com/>. Acesso em: 14 maio 2004.

KLEIN, J.T. et al. Transdisciplinarity: joint problem solving among science, technology, and society - an effective way for managing complexity. Basel: Birkhauser, 2001.

KNORR CETINA, K. Epistemic cultures: how the sciences make knowledge. Cambridge, MA: Harvard University Press, 1999.

KÜFFER, Christoph. [Texto integrante da convocatória da Conferência]. In: HÄBERLI, R. et al. Transdisciplinarity: joint-problem-solving among science, technology and society: Workbook 1: dialogue sessions and idea market. Zurich: Haffmans Sachbuch, 2000. Proceedings of the International Transdisciplinarity 2000 Conference.

KUHN, T. S. A estrutura das revoluções cientificas. São Paulo: Perspectiva, 1975.

LAKATOS, I. História da ciência e suas reconstruções racionais. Lisboa: Edições 70, 1998.

LATOUR, B. From the world of science to that of research? Science, v. 280, n. 5.361, p.208-9, Apr. 1998. Special symposium for the 150th anniversary of the American Association for the Advancement of Science (AAAS).

MANNHEIM, K. The sociological problem of the "intelligentsia". In: Ideology and utopia. San Diego, CA: Harcourt Brace Jovanovich, 1985. 1. ed. 1929.

. The sociology of intellectuals. Theory, Cult. Soc., n.10, p. 369-80, 1993. Publicado pela primeira vez em 1932.

MOODY, J. The structure of a social science collaboration: disciplinary cohesion from 1963 to 1999. American Sociological Review, v. 69, n. 2, p. 213-38, Apr. 2004. 
MORIN, E. Articular os saberes. In: ALVES, N.; GARCIA, R. L.(Org.) O sentido da escola. Rio de Janeiro: DP\&A, 2000.

De la réforme da la université. Bulletin Interactif $d u$ Centre International de Recherches et Études Transdisciplinaires, n. 9-10, févr. 1997. Disponível em: <http://perso.club-internet.fr/nicol/ciret/locarno/loca5c2.htm>. Acesso em: 11 out. 2003.

MUTSCHKE, P.; HAASE, A. Q. Collaboration and cognitive structures in social science research fields: towards socio-cognitive analysis in information systems. Scientometrics, v. 52, n. 3, p. 487-502, 2001.

NICOLESCU, B. La transdisciplinarité: manifeste. Paris: Editions du Rocher, 1996.

NOWOTNY, H. The place of people in our knowledge: towards local objectivity and socially robust knowledge. European Review, v. 7, n. 2, p. 247-262, 1999. Disponível em: $<$ http://www.wiss.ethz.ch/pub/helga $>$. Acesso em: 18 mar. 2004.

POMBO, O. Epistemologia da interdisciplinaridade. In: PIMENTA, Carlos (Coord.). Interdisciplinaridade, humanismo, universidade. Porto: Campo das Letras, 2004. Disponível em: <http://www.educ.fc.ul.pt/docentes/opombo/investigacao/pontofinal.pdf $>$. Acesso em: 12 nov. 2004.

RAMMERT, W. Two styles of knowing and knowledge regimes: between "explicitation" and "exploration" under conditions of "funtional specialization" or "fragmental distribution". Berlin: Technical University, Mar. 2004. (Technical University Technology Studies, Working Papers, TUTS-WP-3-2004).

SANZ-MENÉNDEZ, L. (Org.). Interdisciplinarity as multidimensional concept: its measure in three different research areas. Research Evaluation, v. 10, n.1, p. 39-50, Apr. 2001. Disponível em: <http://www.iesam.csic.es/doctrab2/dt-0104.htm>. Acesso em: mar. 2005.

SCHUMMER, J. Multidisciplinarity, interdisciplinarity, and research collaboration in nanoscience and nanotechnology. Scientometrics, v. 52, n. 3, 487-502, 2001.

SERRES, M. Introdução. A rede de comunicação: Penélope. In: A comunicação. Lisboa: Rés, 1985. p .7-14.

STAR, S. L.; GRIESEMER, J. R. Institutional ecology, "translations" and boundary objects: amateurs and professionals in Berkeley's Museum of Vertebrate Zoology, 1907-39. Social Studies of Science, v. 19, p. 387-420, 1989.

TORO, A. Hacia una teoría de la cultura de la "hibridez" como sistema científico transrelacional, "transversal" y "transmedial. Nuevo Texto Crítico: estudios literários \& estudios culturales. Palo Alto: Stanford University, 25/26, p. 275-329, 2004.

VELHO, L. Cooperação em ciência e tecnologia no Mercosul: síntese final. Brasília: Ministério da Ciência e Tecnologia; Washington: Organização dos Estados Americanos, 1997.

WALLERSTEIN, I. What are we bounding, and whom, when we bound social research. Social Research, n. 6, no.4, p.839-56, 1995. 
WELLMAN, B. Structural analysis: from method and metaphor to theory and substance. In: WELLMAN, B.; BERKOWITZ, S.D. (Ed.). Social structures a network approach. Cambridge: Cambridge University Press, $1988 . \quad$ Disponível em: <www.chass.utoronto.ca/ $\sim$ wellman/publications $>$. Acesso em: 18 set. 2002.

WELSCH, W. Rationality and reason today. In: GORDON, Dane R.; NIZNIK, Józef (Ed.). Criticism and defense of rationality in contemporary philosophy. Amsterdam: Rodopi, 1998. p. 17-31.

Transculturality: the puzzling form of cultures today. In: FEATHERSTONE, Mike; LASH, Scott (Ed.). Spaces of culture: city, nation, world. London: Sage, 1999. p. 194-213.

WITTGENSTEIN, L. Investigações filosóficas. Petrópolis: Vozes, 1996.

XIGAUD, X. A filosofia de Husserl. Buenos Aires: Troquel, 1976. 